\title{
Year of Last Study Visit Attended
}

National Cancer Institute

\section{Source}

National Cancer Institute. Year of Last Study Visit Attended. NCI Thesaurus. Code C159712.

An indication of the last study visit attended by a study sujbect. 